\title{
Vortex Lattice of a Long Superconducting Wire with a Columnar Defect
}

\author{
Edson Sardella and Rodrigo de Alvarenga Freire \\ Departamento de Física, Faculdade de Ciências \\ Universidade Estadual Paulista \\ Caixa Postal 473, 17033-360, Bauru-SP, Brazil
}

Received on 28 February, 2002

\begin{abstract}
In the present work we study a long superconducting wire with a columnar defect in the presence of an applied magnetic field. The cross section of the cylinder is assumed to be circular. The field is taken uniform and parallel to the cylinder axis. We use the London theory to investigate the vortex lattice inside the wire. Although this theory is valid in the limit of low vortex density, that is, when the nearest neighbor vortex distance is much larger than the coherence length, we can obtain a reasonable qualitative description of lattice properties. We calculate: (1) the vortex lattice structure using the simulated annealing technique; (2) the magnetization curve as a function of the applied field.
\end{abstract}

\section{Introduction}

Recent progresses in nanotechnology have made possible the fabrication of mesoscopic superconducting materials with the size comparable to the coherence length $(\xi)$ and London penetration depth $(\lambda)$. This has motivated both experimental and theoretical physicists to investigate the magnetic properties of these small superconductors. The properties of the vortex lattice, for instance, in superconductors of confined geometries change radically with respect to the behavior in the bulk.

Previous works have been dedicated to the investigation of a superconducting wire[1, 2, 3]. In this paper we extend these studies to the case of a superconducting wire with a columnar defect.

\section{The local magnetic field}

The geometry of the problem we will consider in this work is illustrated in Fig. 1. The applied magnetic field $H$ is parallel to the cylinder axis and is denoted by $H$. The internal and external radius of the cylinder are $a$ and $b$ respectively.

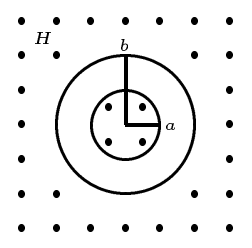

Figure 1. Cross section of a long superconducting cylinder with a columnar defect. The dots represent the applied magnetic field which is directed along the cylinder axis.

The starting point of our study is the London equation for the local magnetic field of a very strong type-II superconductor for which the Ginzburg-Landau parameter $\kappa=\frac{\lambda}{\xi} \gg 1$. For our purposes, this equation can be more conveniently written in cylindrical coordinates as

$-\lambda^{2}\left(\frac{\partial^{2} h}{\partial \rho^{2}}+\frac{1}{\rho} \frac{\partial h}{\partial \rho}+\frac{1}{\rho^{2}} \frac{\partial^{2} h}{\partial \varphi^{2}}\right)+h=\Phi_{0} \sum_{i=1}^{N} \delta\left(\rho-\boldsymbol{\rho}_{i}\right)$

where $\boldsymbol{\rho}_{i}$ is the position of the $i$-vortex line.

This is a Dirichlet problem in which the boundary conditions are given by

$$
h(a, \varphi)=h(b, \varphi)=H .
$$

The solution of the London equation under these boundary conditions may be found by means of the Green's function method. For the local magnetic field, we have 
where

$$
h(\rho, \varphi)=\Phi_{0} \sum_{i=1}^{N} G\left(\rho, \varphi, \rho_{i}, \varphi_{i}\right)+h_{1}(\rho)
$$

The solution contains two distinct parts[4]. The first term is a particular solution of the London equation as if no applied field were present. The second term is the solution of the homogeneous London equation in the absence of any vortex line (Meissner state).

The Green's function $G\left(\left(\rho, \varphi, \rho^{\prime}, \varphi^{\prime}\right)\right.$ has the form

$$
G\left(\rho, \varphi, \rho^{\prime}, \varphi^{\prime}\right)=\frac{1}{2 \pi} \sum_{m=-\infty}^{\infty} e^{i m\left(\varphi-\varphi^{\prime}\right)} g_{m}\left(\rho, \rho^{\prime}\right)
$$

where the Fourier coefficients are given by

$$
g_{m}\left(\rho, \rho^{\prime}\right)= \begin{cases}\frac{\Delta_{m}\left(\rho^{\prime}, b\right) \Delta_{m}(a, \rho)}{\lambda^{2} \Delta_{m}(a, b)}, & a \leq \rho \leq \rho^{\prime} \\ \frac{\Delta_{m}\left(\rho^{\prime}, a\right) \Delta_{m}(b, \rho)}{\lambda^{2} \Delta_{m}(a, b)}, & \rho^{\prime} \leq \rho \leq b\end{cases}
$$

$$
\Delta_{m}(x, y)=K_{m}(x / \lambda) I_{m}(y / \lambda)-K_{m}(y / \lambda) I_{m}(x / \lambda) .
$$

Here $K_{m}(x)$ and $I_{m}(x)$ are the Bessel functions of second kind.

The homogeneous solution can be easily found by using standard methods of solution of differential equations. One has

$$
h_{1}(\rho)=H\left[\frac{\Delta_{0}(a, \rho)-\Delta_{0}(b, \rho)}{\Delta_{0}(a, b)}\right] .
$$

\section{The free energy}

We will move forward on the determination of the London free energy (the Helmholtz free energy in the thermodynamic context). In the London approximation this energy (per unit volume) is given by

$$
\mathcal{F}=\frac{1}{8 \pi A} \int_{0}^{2 \pi} \int_{a}^{b}\left[\frac{\lambda^{2}}{\rho^{2}}\left(\frac{\partial h}{\partial \varphi}\right)^{2}+\lambda^{2}\left(\frac{\partial h}{\partial \rho}\right)^{2}+h^{2}\right] \rho d \rho d \varphi
$$

where $A=\pi\left(b^{2}-a^{2}\right)$ is the area of the cross section of the cylindrical shell.

The London free energy $\mathcal{F}$ is a thermodynamic function of the magnetic induction $B$, which is inconvenient for calculations involving a fixed applied magnetic field. Therefore, it is necessary to use a Legendre transformation for the Gibbs free energy

$$
\mathcal{G}=\mathcal{F}-\frac{B H}{4 \pi}
$$

where $B$ is the magnetic induction which is defined as a spatial average of the local magnetic field

$$
B=\frac{1}{A} \int h d^{2} \rho
$$

By means of these definitions of $\mathcal{F}$ and $B$, after a tedious but straightforward calculation, we find for the Gibbs free energy

$$
\begin{aligned}
\mathcal{G}= & \frac{\Phi_{0}^{2}}{8 \pi A} \sum_{i, j=1}^{N} G\left(\rho_{i}, \varphi_{i}, \rho_{j}, \varphi_{j}\right)+\frac{\Phi_{0} H}{4 \pi A} \sum_{i=1}^{N} \frac{\Delta_{0}\left(a, \rho_{i}\right)-\Delta_{0}\left(b, \rho_{i}\right)}{\Delta_{0}(a, b)} \\
& -N \frac{\Phi_{0} H}{4 \pi A}-\frac{\lambda^{2} H^{2}}{4 A \Delta_{0}(a, b)}\left[\frac{b}{\lambda} \delta_{0}(a, b)+\frac{a}{\lambda} \delta_{0}(b, a)-2\right],
\end{aligned}
$$


where we used th following definition

$$
\delta_{m}(x, y)=K_{m}(x) I_{m+1}(y)+K_{m+1}(y) I_{m}(x) .
$$

\section{The limit of a thin wire}

We will now take the limit of a very thin superconducting wire with a columnar deffect. We take both $a \ll \lambda$ and $b \ll \lambda$. The following results are also valid for both a small disc (if $a=0$ ) and a small ring (if $a \neq 0)[3]$. Within this limit the local magnetic field and the Gibbs free energy are enormously simplified. For the local magnetic field we obtain

$$
\begin{aligned}
& \bar{h}(\rho, \varphi)=h(\rho, \varphi)-H= \\
& =\frac{\Phi_{0}}{2 \pi \lambda^{2}} \sum_{j=1}^{N}\left[\ln \frac{\left|1-Z \overline{Z_{j}}\right|\left|1-\frac{a^{2}}{b^{2} Z_{j}}\right|}{\left|Z-Z_{j}\right|\left|1-\frac{a^{2} \overline{Z_{j}}}{b^{2} \bar{Z}}\right|\left|1-\frac{a^{2} Z}{b^{2} Z_{j}}\right|}-\frac{\ln (|Z|) \ln \left(\left|Z_{j}\right|\right)}{\ln (b / a)}\right] \\
& +\frac{H b^{2}}{4 \lambda^{2}}\left[|Z|^{2}-1-\frac{\ln |Z|}{\ln (b / a)}\left(1-\left(\frac{a}{b}\right)^{2}\right)\right]
\end{aligned}
$$

where we have used the complex notation $Z=\frac{\rho}{b} e^{i \varphi}$.

For the Gibbs free energy we find

$$
\begin{aligned}
\mathcal{G}_{N}-\mathcal{G}_{0}= & \frac{\Phi_{0}^{2}}{16 \pi^{2} A \lambda^{2}} \sum_{i, j=1}^{N}\left[\ln \frac{\left|1-Z_{i} \overline{Z_{j}}\right|\left|1-\frac{a^{2}}{b^{2} Z_{j} \bar{Z}_{i}}\right|}{\left|Z_{i}-Z_{j}\right|\left|1-\frac{a^{2} \overline{Z_{j}}}{b^{2} \overline{Z_{i}}}\right|\left|1-\frac{a^{2} Z_{i}}{b^{2} Z_{j}}\right|}-\frac{\ln \left(\left|Z_{i}\right|\right) \ln \left(\left|Z_{j}\right|\right)}{\ln (b / a)}\right] \\
& +\frac{\Phi_{0} H b^{2}}{16 \pi A \lambda^{2}} \sum_{i=1}^{N}\left[\left|Z_{i}\right|^{2}-1-\frac{\ln \left|Z_{i}\right|}{\ln (b / a)}\left(1-\left(\frac{a}{b}\right)^{2}\right)\right]
\end{aligned}
$$

where $\mathcal{G}_{0}$ is the Gibbs free energy in the Meissner state. These results are in agreement with those of [3] in the particular case $a=0$.

\section{Results and Discussion}

In the mixed state the magnetization shows a series of picks as a function of the applied magnetic field. Each pick is generally interpreted as an indication of the entrance of an individual vortex in the sample. Soon after the entrance of a vortex, they rearrange themselves in order to lower the Gibbs free energy. This configuration corresponds to the global optimum. Each entrance of a vortex is associated with a critical field, the so called matching field. These fields were found as follows. As we have stated previously, the vortices penetrate one at the time. At the transition from $N$ to $N+1$ vortices, we assume that the Gibbs free energy is continuous, that is, $\mathcal{G}_{N}(H)=\mathcal{G}_{N+1}(H)$. The solution of this equation provides the value of the matching field. Full details of how to determine these matching fields can be found in Ref. [2].

The matching fields were determined up to $N=24$ vortices. Next, we calculated numerically the magneti- zation $4 \pi M=B-H$. The result is depicted in Fig. 2.

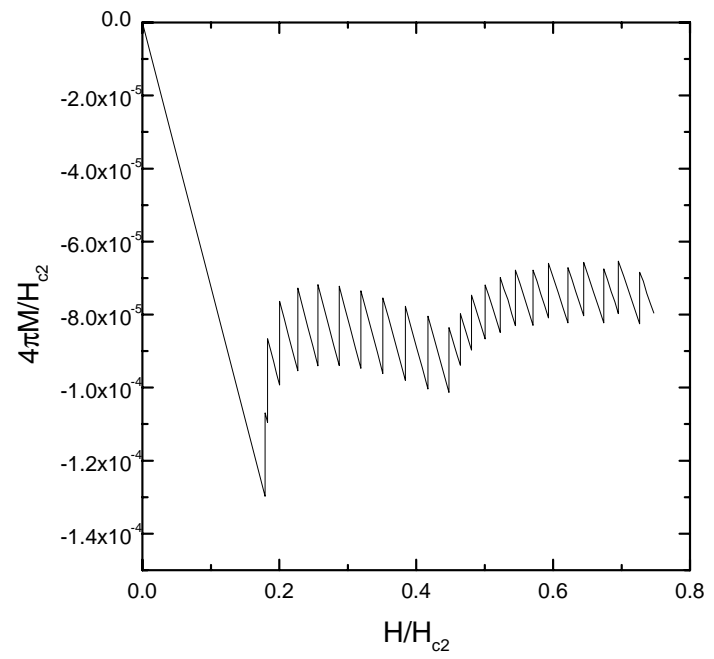

Figure 2. Magnetization versus the applied field for $\kappa=100$, $a=\xi$, and $b=10 \xi$. The fields are in units of the upper critical field $H_{c 2}$.

As can be seen in this figure, the magnetization changes in small steps. Each pick signals the penetration of a vortex line. In addition, the magnetization 
remains negative for all values of $H$ until $N=24$. Recently, some authors $[3,5]$ suggested that for confined geometries of dimensions comparable to the fundamental lengths $(\lambda, \xi)$, the magnetization presents a positive sign. This indicates that the vortex system may be in a paramagnetic Meissner state. This has been interpreted as a manifestation of either a metastability $[3,5]$ of the vortex system or the formation of a giant vortex state[6]. Within our approach, we will never be able to detect the formation these vortex states. First, we cannot find giant vortex states due to a limitation of London theory which supposes that the nearest neighbor distances between the vortices is much larger than the coherence length $\xi$. Second, we cannot find metastability since we use the simulated annealing method whic rarely stops at a local minimum of the vortex state.

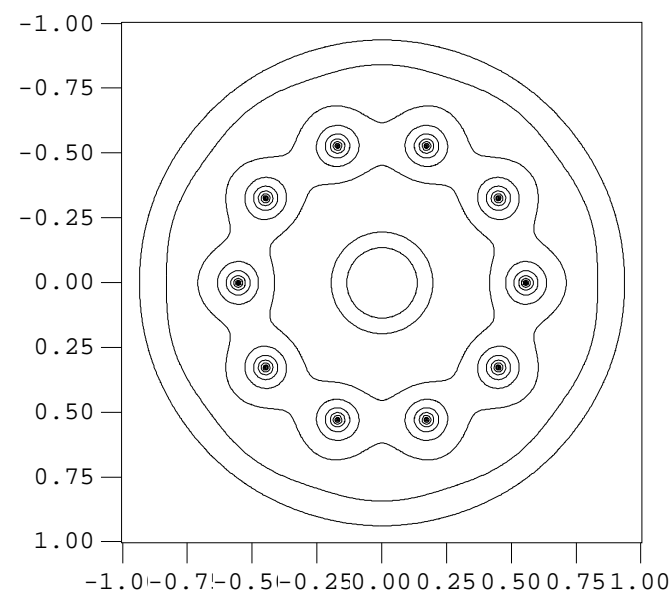

Figure 3. Distribution of the local magnetic field $\bar{h}(\rho, \varphi)$ for $N=10$ vortices. The lengths are in units of $b$. The darker regions indicate where the field is more intense.

We also determined the distribution of the local magnetic field for two values of $N$. As we can see in Fig. 3, the vortices are distributed in a ring around the columnar deffect. However, for $N=11$ this single ring breaks into two rings (see Fig. 4). This jump from a regime of a single- to a multi-ring vortex state can bo clearly seen in Fig. 2 in form of a pick at $H=0.45 H_{c 2}$

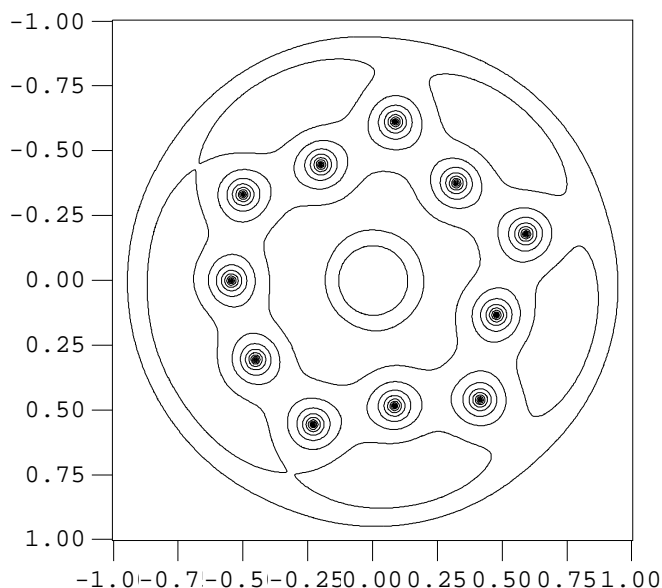

Figure 4. The same as Fig. 3, except that now $N=11$.

\section{Acnowledgement}

We thank the São Paulo Agency FAPESP for financial support.

\section{References}

[1] G. Böbel, Nuovo Cimento 38, 6320 (1965).

[2] P. A. Venegas e Edson Sardella, Phys. Rev. B 58, 5789 (1998).

[3] E. Akkermans, D. M. Gangart, e K. Mallick, Phy. Rev. B 63, 4523 (2001).

[4] Detailed solution of the London equation for the present geometry will be given elsewhere.

[5] A. K. Geim, S. V. Dubonos, J. G. S. Lok, M. Henini, e J. C. Maan, Nature, 396, 144 (1998).

[6] B. J. Baelus at al., Phys. Rev. B 63, 144517 (2001). 